\title{
Obesidade: uma perspectiva plural
}

\author{
Obesity: a plural perspective
}

Emanuela NogueiraWanderley ${ }^{1}$

Vanessa Alves Ferreira ${ }^{1}$

${ }^{1}$ Departamento de N utrição, Universidade Federal dos Vales do Jequitinhonhae Mucuri. Rua da Glória 187, Centro. 39100-000 Diamantina M G. nutri.manu@hotmail.com
Abstract 0 besity is a disease characterized by the excessive accumulation of corporal fat, which produces del eterious effects to the health. There is consent in the literature that its etiology is multifactorial, involving biological, historical, ecological, political, social-economical, psychosocial and cultural aspects. In that sense, this article's objective is to talk about the multi-factorial character of obesity, involving the wide variety of environmental and genetic factors implicated in its etiology, starting from secondary studies of literature revision in the main bases of data and specialized libraries. N owadays, obesity has been considered the most important nutritional disorder in the developed countries and the onesin development, due to the increase of its incidence. The approach of the genetic, metabolic, psychosocial, symbolic, and cultural aspects in addition to the lifestyle allowed to base obesity as a plural illness, and showed the necessity to create public politics with multidiscipline and inter-sectorial actions, that value the partnership between government and civil society, in the prevention and combat of obesity and the promotion of health, making possible the community's participation in that process, through responsibility and self care. Key words O besity, Nutritional transition, Risk factors, Public politics
Resumo A obesidadeéuma doença caracterizada pelo acúmulo excessivo de gordura corporal, que produz efeitos deletérios à saúde. Há um consenso na literatura de que sua etiologia é multifatorial, envolvendo aspectos biológicos, históricos, ecológicos, políticos, socioeconômicos, psicossociais e culturais. N esse sentido, este artigo tem por objetivo abordar o caráter multifatorial da obesidade, envolvendo a ampla variedade de fatores ambientais e genéticos implicados na sua etiologia a partir de estudos secundários provenientes do trabalho de revisão da literatura nas principais bases de dados e bibliotecas especializadas. A tualmente, a obesidade tem sido considerada a mais importante desordem nutricional nos países desenvolvidos e em desenvolvimento, devido ao aumento da sua incidência. A abordagem dos aspectos genéticos, metabólicos, psicossociais, simbólicos, culturais e de estilo de vida permitiu fundamentar a obesidade enquanto uma enfermidade plural e a necessidade de criar políticas públicas com ações multidisciplinares e intersetoriais, que val orizem a parceria entre governo e sociedade civil, na prevenção e combate à obesidade e promoção da saúde, possi bilitando a parti cipação da comunidade nesse processo, através da responsabilização e do autocuidado.

Palavras-chave O besidade, Transição nutricional, Fatores de risco, Políticas públicas 


\section{Introdução}

A obesidadeé uma doença caracterizada pelo acúmulo excessivo de gordura corporal ${ }^{1} \mathrm{em}$ um nível que compromete a saúde dos indivíduos, acarretando prejuízos tais como alterações me tabólicas, dificuldades respiratórias e do apareIho locomotor ${ }^{2,3}$. Além de se constituir enquanto fator de risco para enfermidades tais como dislipidemias, doenças cardiovasculares, diabetes melito tipo II e al guns tipos de câncer ${ }^{2,4,5}$.

O diagnóstico da obesidadeérealizado a partir do parâmetro estipulado pela Organização Mundial deSaúde ${ }^{1}$ o body mass index (BM I) ou índice de massa corporal (IMC), obtido a partir da relação entre peso corpóreo $(\mathrm{kg})$ e estatura $(\mathrm{m})^{2}$ dosindivíduos. A través deste parâmetro, são considerados obesos os indivíduos cujo IMC encontra-se num valor igual ou superior a $30 \mathrm{~kg} / \mathrm{m}^{2}$.

$\mathrm{Na}$ literatura, existe um consenso de que a etiologia da obesidade é bastante complexa, apresentando um caráter multifatorial ${ }^{1,6-9}$. Envolve, portanto, uma gama de fatores, incluindo os históricos, ecológicos, políticos, socioeconômicos, psicossociais, biológicos e culturai $5^{8-10}$. Ainda assim, nota-se que, em geral, os fatores mais estudados da obesidade são os biológicos relacionados ao estilo devida, especialmenteno quediz respeito ao binômio dieta eatividadefísica. Tais investigações se concentram nas questões relacionadas ao maior aporte energético da dieta ${ }^{4,5,9,11}$ e na redução da prática da atividade física com a incorporação do sedentarismo, configurando o denominado estilo de vida ocidental contemporâneo ${ }^{4,9,11}$.

Nesse sentido, este artigo tem por objetivo abordar o caráter multifatorial da obesidade envolvendo a ampla variedade de fatores ambientais e genéticos implicados na sua etiologia a partir deestudos secundários provenientes do trabaIho de revisão da literatura nas principais bases de dados e bibliotecas especializadas. Frente à amplitude do tema, nosso intuito é apontar de forma atualizada os principais fatores envolvidos na etiologia da obesidade por reconhecer a importância do estudo acerca da enfermidade dentro de uma perspectiva plural. A nosso ver, reduzir as investigações somente ao aspecto biológico ou social da doença limita o entendimento e 0 alcance explicativo dessa problemática.

\section{A emergência da obesidade}

Atualmente, a obesidade tem sido considerada a mais importante desordem nutricional nos paí- ses desenvolvidos e em desenvolvimento, devido ao aumento da sua incidência ${ }^{1}$. De acordo com os dados da O rganização M undial de Saúde', esse agravo possivelmente atinge $10 \%$ da população destes países. N os países da América, a obesidade vem aumentando, para ambos os gêneros, tanto em países desenvolvidos quanto nas sociedades em desenvolvimento. $\mathrm{Na}$ Europa, verificou-senum decênio um incremento entre $10 \%$ a $40 \%$ da obesidade na maioria dos países. Na região O este do Pacífico, compreendendo a Austrália, o Japão, Samoa e China, também nota-se a elevação da prevalência da obesidade. No entanto, a China e o Japão, apesar do aumento da obesidade em comparação com outros países desenvolvidos, apresentam as menores prevalências mundiais. N os continentes africano e asiático, a obesidade é ainda relativamente incomum, sendo que sua prevalência é mais el evada na população urbana em relação à população rural. $M$ as nas regiões economicamente avançadas destes continentes, a prevalência pode ser tão alta quanto nos países desenvolvidos ${ }^{1}$.

No Brasil, a análise de quatro estudos de base populacional realizados no país - Estudo Nacional sobre Despesas Familiares (EN DEF), realizado entre 1974-1975; a Pesquisa Nacional sobre Saúde e Nutrição (PNSN), de 1989; a Pesquisa sobre Padrões de Vida (PPV), desenvolvida em 1996-19974; e a Pesquisa de Orçamentos Famili$\operatorname{ares}^{12}$ (POF), de 2002-2003 - permitiu avaliar a magnitude dos agravos nutricionais mais relevantes, incluindo a emergência da obesidade e verificar seus principais determinantes, assim como traçar a ten dência do comportamento desses agravos no país. De acordo com esses estudos, a prevalência da desnutrição em crianças e adultos teve um declínio acelerado nas últimas décadas, enquanto o sobrepeso e a obesidade aumentaram na população brasileira, principalmente entre os adultos.

Os resultados obtidos no ENDEF de 1974/75 permitiram verificar que a obesidade excedeu a desnutrição somenteentre os adultos dealta renda, ao passo que na PNSN de 1989, constatou-se que a obesidade superou a desnutrição entre os homens de renda alta e média e entre as mulheres de todos os níveis de renda ${ }^{13}$.

Segundo Batista Filho e Rissin ${ }^{13}$, as diferenças geográficas no país expressam diferenciações sociais na distribuição da obesidade. Inicialmente, verificou-se maior prevalência de excesso de peso nas regiões mais desenvolvidas (Sul, Sudeste e Centro-O este) do país e nos estratos de renda mais elevados, mas já observa-se tendência de 
aumento da obesidade nas regiões $\mathrm{N}$ orte e N ordeste e nos estratos de renda mais baixos. Assim, a comparação dos resultados dos estudos referidos acima nas regiões N orte e N ordeste com os das regiões Sul, Sudestee Centro-O este permitiu assinalar a maior prevalência da obesidade nas últimas regiões citadas. Além disso, os estudos indicaram que a ocorrência desse agravo praticamente triplicou entrehomens emulheres maiores de vinte anos na região N ordeste e somente entre os homens do Sudeste ${ }^{13}$.

Dados recentes da POF 2002/03'12 revelaram que cerca de $40 \%$ dos adultos no Brasil estão com excesso de peso, e que 8,9\% dos homens e $13,1 \%$ das mulheres são obesos. N as mulheres, a ocorrência mais el evada de excesso de peso éencontrada nos estratos de menor renda ${ }^{2,9,12}$. Observando-se ainda estabilidade e tendência de declínio nos segmentos de elevada renda, com exceção da região N ordeste, na qual a obesidade continua emergindo. A maior concentração de mulheres com excesso de peso é observada nas áreas rurais de todo o país, situação diferente apenas no N ordeste, onde a maior concentração se dá nas áreas urbanas. Já nos homens, as maiores prevalências são observadas nas áreas urbanas das regiões Sudeste, Sul e Centro-O este e nos estratos de maior renda ${ }^{12}$.

A compreensão do comportamento da obesidade no Brasil mostra-se essencial para a definição de prioridades e estratégias de ações em saúde pública. Dessa forma, é necessário que se jam incorporadas ações direcionadas para a prevenção e controle desse agravo, assumindo destaque as medidas de educação em saúde e nutrição em âmbito nacional, assim como em todos os segmentos da sociedade. N este sentido, a garantia dos principais mecanismos de preven ção/ interven ção da obesidade deve ser assegurada a todos osindivíduos, incluindo a aquisição de uma dieta digna sob o ponto de vista qualitativo e quantitativo, a prática deatividade física de lazer orientada e a assistência multiprofissional para todos os indivíduos.

\section{M etodologia}

A estratégia de busca de artigos incluiu pesquisa nas bases eletrônicas M ED LIN E (N ational Library of M edicine, Estados Unidos), LILACS (Literatura Latino-americana e do Caribe em Ciências da Saúde), bibliotecas especializadas tais como a da Escola Nacional deSaúde Pública Sérgio Arouca (EN SP) eda Universidade de São Paulo (USP).
As palavras-chaves "obesidade", "transição nutricional", "epidemiologia", "etiologia", "diagnóstico", "genética", "hormônios", "fatores endócrinos", "mídia", "comportamento alimentar", "aspectos sociais", "renda", "cultura", "aspectos simbólicos", "aspectos psicológicos" foram utilizadas como descritores. As referências bibliográficas dos estudos assim localizados foram também rastreadas para localizar outros trabalhos pertinentes ao assunto abordado nesse artigo. 0 levantamento bibliográfico totalizou 123 referências, das quais 55 foram selecionadas. E incluíram 4 livros, 9 capítulos de livros, 35 artigos, 2 dissertações e 5 publicações de instituições (WH O, ABESO, MS, OPAS, IBGE). A seguir, serão enfocados os principais fatores relacionados à etiologia da obesidade de acordo com a literatura pesquisada dentro de uma abordagem plural.

\section{Influências históricaseecológicas}

A ascensão da obesidade no mundo pode ser compreendida enquanto resultante do fenômeno da transição nutricional. Esta dinâmica caracteriza-se pela modificação nos padrões de distribuição dos agravos nutricionais de uma dada população no tempo ${ }^{4,14}$, ou seja, uma redução na prevalência das doenças atribuídas ao subdesenvolvimento e, contrariamente, ao aumento daquelas doenças vinculadas à modernidade, sendo, em geral, uma passagem da desnutrição para a obesidade ${ }^{4}$. Esse processo tem como determinantes as mu danças quevêm ocorrendo nos padrões de alimentação e de atividade física das populações $5^{4,14}$, e que, segundo Popkin et al. ${ }^{15}$, se correlacionam com mudanças econômicas, sociais, demográficas e relacionadas à saúde decorrentes do processo de modernização mundial.

Para Fischler ${ }^{16}$, a modernização das sociedades desencadeou a reordenação do contexto de vida do homem contemporâneo e fez emergir um novo modo de vida, no qual a oferta e 0 consumo dealimentos aumentou expressivamente e todo tipo de gênero tornou-se acessível, notadamente devido ao desenvolvimento detecnologia alimentar.

As modificações na alimentação referem-seà crescente incorporação pela população da denominada "dieta ocidental" ou "dieta moderna". Esta pode ser caracterizada como uma dieta rica em gordura (principalmente as de origem animal), açúcares ealimentos refinados. E, em contrapartida, pela quantidade reduzida de fibras e outros carboidratos complexos ${ }^{8,14,15}$. Por outro lado, a 
redução da atividadefísica ea adesão ao estilo de vida sedentário devem-se a alterações na esfera do trabalho, do lazer e do modo de vida moderno. Estas alterações, por sua vez, estão associadas ao processo de desenvolvimento e modernização das sociedades contemporâneas, 13,14.

No Brasil, também verificaram-se mudanças na dieta e na ati vidade física da população. 0 estudo de M ondini e M onteiro ${ }^{14}$ constatou modificações no padrão de alimentação da população brasileira entre os anos de 1961/63, 1975/76 e $1987 / 88$. As modificações observadas referem-se ao menor aporte de carboidratos no consumo energético total e sua substituição por gorduras, principalmenteas de origem vegetal em detrimento das gorduras de origem animal, o consumo excessiv vo deaçúcar einsuficientede carboidratos complexos e fibras ${ }^{14,17}$.

No que diz respeito à atividade física, ainda segundo esses autores, houve transformações na estrutura das ocupações de trabalho. O bserva-se nas últimas décadas à mecanização do processo de trabal ho e o aumento da participação da população no setor terciário da economia, cujas ocupações requerem menor demanda energética que nos demais setores, tais como o secundário e primário. E a redução nas atividades do setor primário. Tal situação relaciona-se ao fenômeno da transição demográfica, com a concentração da população nas áreas urbanas do país e, ainda, em decorrência do desenvolvimento tecnológico $0^{13,14}$.

As considerações acima permitem inferir que, conforme afirmaram M onteiro et al ${ }^{14}$, , o país vem substituindo o problema de escassez alimentar pelo excesso dietético. Entretanto, torna-seoportuno ressaltar que, apesar da ascensão da obesidade no país, agravos relacionados à carência de alimentos, tais como a desnutrição e as anemias carenciais ainda são relevantes, especial mentenos estratos de menor renda e em regiões de menor desenvolvimento socioeconômico tal como aregião nordeste do país. Esse paradigma é uma característica marcante da transição nutricional no Brasi| $8,13,17,18$.

Neste sentido, dados recentes da Pesquisa de Orçamentos Familiares 2002-2003 ${ }^{12}$, realizada pelo IBGE em parceria com o M inistério da Saúde no Brasil, confirmam essa tendência do incremento no consumo de açúcares e gorduras na população brasileira. Mais adiante, abordaremos mais detalhadamente os resultados desta pesquisa.
Fatores genéticos e metabólicos da obesidade

Os aspectos biológicos da obesidade envolvendo a genética e o metabolismo têm sido discutidos em vários trabalhos ${ }^{5,19-21}$. Nesta direção, a teoria da economia energética vem sendo apontada como possível contribuinte para o desenvolvimento da obesidade. Segundo esta, em situações de adversidades biológicas esociais em quehá déficit de energia, o organismo aciona uma série de mecanismos metabólicos adaptativos, que visa promover a redução no gasto energético como estratégia de sobrevivência. Essa adaptação leva o organismo a um novo ponto de equilíbrio, em que o gasto e a ingestão energética são inferiores ao normal. Estenovo equilíbrio, contudo, revelasefrágil, e um aumento na ingestão de alimentos podelevar a um ganho de peso, consequência do aumento da eficiência metabólica adquirida5,8,22.

Francischi et al. ${ }^{21}$ destacam em sua revisão que há forte influência genética no desenvolvimento da obesidade, mas seus mecanismos ainda não estão totalmente esclarecidos. M as já se reconhece que 0 apetite e o comportamento alimentar sofrem influência genética e há indícios de que 0 componentegenético atue sobre o gasto energético, notadamente sobre a taxa metabólica basal $(T M B)^{8,21,23}$. Desordens endócrinas como o hipotireoidismo e problemas no hipotálamo, alterações no metabolismo de corticosteróides, hipogonadismo em homens e ovariectomia em mulheres, síndrome de Cushing e síndrome dos ovários policísticos podem ainda conduzir à obesidade $21,24-26$.

0 peso corporal é regulado por uma interação complexa entre hormônios e neuropeptídeos, sob o controle principal de núcleos hipotalâmicos ${ }^{24}$. Mutações nos genes dehormônios eneuropeptídeos, de seus receptores ou de seus elementos regulatórios, têm sido associadas à obesidade ${ }^{24,26}$, embora não explicando as formas mais comuns dessa patologia ${ }^{24}$.

N este sentido, M arques-Lopes et al. ${ }^{23}$ relatam em sua revisão que os genes intervêm na manutenção de peso egordura corporal estáveis ao longo do tempo. Essa influência advém da participação desses no controle de vias eferentes (leptina, nutrientes, sinais nervosos, entre outros), demecanismos centrais (neurotransmissores hipotalâmicos) e de vias aferentes (insulina, catecolami- 
nas, sistema nervoso autônomo). A leptina é um hormônio secretado pelo tecido adiposo subcutâneo ${ }^{27}$ em resposta ao armazenamento de gordura ou ao excesso de ingestão alimentar, sendo um importante marcador da quantidade de tecido adiposo ${ }^{24,27}$. De acordo com Fonseca-Alaniz et al. ${ }^{27}$, a leptina reduz 0 apetite por inibir a formação de neuropeptídeos relacionados ao apetite, como o neuropeptídeo Y, e por promover o aumento da expressão de neuropeptídeos anorexígenos, como o hormônio estimulante de a-melanócito (a-M SH) eo hormônio liberador de corticotropina ( $\mathrm{CRH})$. A deficiência congênita desse hormônio é responsável por 1 a $2 \%$ dos casos de obesidade mórbida precoce ${ }^{24}$.

Outros fatores envolvidos na gênese da obesidade são a colecistocinina (CCK), a ghrelina, o neuropeptídeo Y (NPY) e o peptídeo YY, que são sustâncias envolvidas no controle da ingestão al imentar ${ }^{24,26}$. A CCK e o peptídeo YY são liberados pelo trato gastrointestinal e ao nível cerebral inibem a ingestão alimentar, promovendo a sacie dade após uma refeição. O N PY é sintetizado no SNC e estimula a ingestão. A redução nos níveis de insulina e leptina ativa os neurônios produtores de NPY no hipotálamo, e a leptina inibe sua síntese. A ghrelina é um hormônio gastrointestinal estimulador do apetite e faz parte dos sistemas de regulação do peso corporal. A produção excessiva de ghrelina podelevar à obesidade. Alterações no controle da liberação dessas e de outra sustâncias envolvidas na regulação do balanço energético, assim provocando disfunções nos sistemas de regulação do peso corporal por retroalimentação, podem ocasionar a obesidade ${ }^{24}$.

Os aspectos genéticos e metabólicos abordados são apenas uma parcela diminuta da ampla variedade desses aspectos que exercem influência na etiologia da obesidade, e que merecem maior atenção dos pesquisadores para que haja a elucidação dos vários mecanismos pelos quais eles influenciam no desenvolvimento dessa patologia.

\section{Aspectos socioculturais esimbólicos}

A obesidade enquanto um atributo físico é percebida, interpretada e influenciada pelo sistema social ${ }^{8,28}$. Portanto, valores socioculturais relacionados à obesidade podem variar de uma sociedade para outra, nos diferentes contextos históricos $^{8}$. Assim, a idéia de saúdequeanteriormente esteve associada à corpulência, hoje tem seu significado modificado com as novas concepções e crenças culturais acerca do corpo magro $8,29-31$.
0 corpo assume um aspecto existencial fundamental porque é por intermédio dele que os sujeitos se manifestam no mundo ${ }^{32}$. Os cuidados corporais relacionados à beleza, à estética eà saúde são regidos por um sistema de regras sociais fruto da relação entre os indivíduos, seu corpo ea sociedade. Existe, portanto, um sistema de códigos para viver um corpo que é interiorizado e manifestado por um determinado grupo soci$a^{33}$. Na contemporaneidade, observam-se padrões corporais próprios relacionados ao corpo magro, delineado, estético e eternamente jovem. O corpo desejado hoje é o funcional, por vincular-se a símbolos de beleza, realização pessoal e erotismo ${ }^{28}$. Para Baudrillard ${ }^{34}$, as estruturas atuais de produção e consumo induzem à representação do corpo como capital, do corpo enquanto objeto de troca. Neste sentido, a higiene, a moda, os cosméticos, a estética, os diferentes tratamentos corporais, as várias modalidades esportivas e o erotismo são recursos rentáveis para al cançar um padrão de corpo vigente.

Carvalho e Martins $s^{35}$ propõem que a obesidade éum estado destoante dos padrões de normalidade na cultura, ou seja, o indivíduo obeso é anormal porque difere do ideal de beleza do corpo magro e/ou musculoso construído pela sociedade. $\mathrm{M}$ as também pode ser compreendida como um estado patológico por gerar impotência do corpo e minimizar as possibilidades de vida do indivíduo no seu ambiente.

Nas sociedades modernas, nota-se que a rejeição às pessoas obesas é progressivamente maior ${ }^{30,31}$, mas em algumas sociedades, como determinados grupos e tribos africanas, uma rotundidade corporal razoável ainda está associada ao sucesso econômico, força política, condição social, símbolo de beleza e maternidade ${ }^{29,31}$, assim como era nos países desenvolvidos até 0 século $X I X^{30}$. Padrões corporais relacionados à obesidade podem, portanto, variar de sociedade para sociedade nos diferentes contextos e grupos sociais ${ }^{29}$.

Em geral, observa-se que dentro da esfera social a obesidade recebe duas definições: a de um estado desviante dos padrões de normalidade na cultura. Assim, o corpo gordo é considerado fora da norma social vigente por que contrapõe o modelo de corpo magro e/ou musculoso tido como socialmente aceitável. A outra definição compreende a obesidade enquanto um estado patológico, uma doença, em função dos inúmeros sinais e sintomas da enfermidade, da sua alta relação com outras comorbidades e ainda por comprometer a qualidade de vida do indivíduo no seu ambiente ${ }^{35}$. 


\section{Os fatores psíquicos da obesidade}

Segal ${ }^{36}$ acredita que, de todos os fatores envolvidos na obesidade, os psicológicos e psiquiátricos possuem maior relevância, não tanto pelo seu papel etiológico edeprognóstico, massim por seu papel histórico. Nas primeiras seis décadas do século $X X$, a obesidade era entendida como resultante de déficits morais e/ou problemas psíquicos.

Os indivíduos obesos eram vistos como os únicos culpados pela sua condição, ou seja, são gordos por que são incapazes de se controlar ${ }^{16}$. Além disso, eram percebidos como tendo baixa auto-estima, limitações intelectuais, mau funcionamento mental, tais como excesso de ansiedade e covardia (por utilizarem a gordura para se esconder), e egoístas por comerem mais do que os outros indivíduos ${ }^{36}$. Os obesos são assim considerados transgressores, ou seja, violam constantemente as regras e normas sociais sobre 0 comer e a comida ${ }^{16,35}$.

Almeida et al. ${ }^{37}$ relatam que dentre os prejuízos diversos à saúde associados à obesidade estão os aspectos psicológicos, como aqueles relacionados à imagem corporal que envolve diversosfatores queseinterrelacionam, como os emocionais, de atitude e também perceptuais. Um ambiente competitivo, com altas expectativas da adequação a um corpo perfeito, pode criar diversos problemas de realização pessoal para um indivíduo ${ }^{35}$.

As sociedades contemporâneas têm seu comportamento progressivamente modulado pela mídia, que impõe ideais de saúde e juventude, difunde consel hos dietéticos, estéticos, desportivos, eróticos e psicológicos. E que, apesar das diferenças raciais eétnicas, persisteem impor um padrão idealizado ehomogêneo de beleza. Entretanto, o que se vê na mídia enquanto imposição de valores ${ }^{28,38,39}$ pode gerar equívocos, já que esta nos dispõe imagens e padrões pouco prováveis de serem alcançados ${ }^{38}$, eque desrespeitam as necessidades singulares de cada indivíduo, distanciando-o do seu corpo singular, capaz e saudável a seu modo ${ }^{35}$.

A postura atual é a de que a população geral de obesos não apresenta maiores níveis de psicopatologia, quando comparada à população geral não obesa ${ }^{36}$. Entretanto, obesos em tratamento apresentam maiores níveis de sintomas depressivos, ansiedade, transtornos alimentares, de personalidade e distúrbios da imagem corporal. Essa postura pode ser justificada pelo fato de que não é necessária a presença de psicopatologia para 0 aparecimento da obesidade, quea psicopatologia é restrita a grupos específicos ${ }^{36,40}$, e pela obesidade ser vista como causadora da psicopatologia e não como consequência desta última ${ }^{36}$.

Segundo Dobrow et al. ${ }^{41}$, existem subgrupos deindivíduos obesos que possuem padrões anormais de alimentação: aqueles que têm o transtorno da compulsão alimentar periódica (TCAP) e os com a síndrome do comer noturno (SCN). O TCAP é definido como episódios de compulsão alimentar, com ingestão de grandes quantidades de alimentos num curto espaço de tempo, não seguidos de método compensatório. A compulsão alimentar é acompanhada de sentimentos de desconforto físico, angústia, vergonha e culpa ${ }^{42}$. Esse transtorno ocorre em maior proporção na população obesa, embora esteja presente na população com peso normal. Os indivíduos obesos com esse transtorno frequentemente apresentam imagem corporal negativa, sofrem de angústia psicológica como baixa auto-estima, depressão, ansiedade, síndrome do pânico etranstornos de personalidade ${ }^{43,44}$.

0 estudo de Zanella e M atos ${ }^{40}$ revelou elevada prevalência do TCAP e de episódios isolados de compulsão alimentar, sintomas de ansiedade, depressão e preocupação excessiva com a imagem corporal em indivíduos com obesidade grave. Também foi observada associação entre a ocorrência de ansiedade como traço de personalidade e a ocorrência de TCAP, assim como entre a preocu pação excessiva com a imagem corporal e a ocorrência de episódios isolados de compulsão alimentar.

A SCN é um transtorno com três características básica: anorexia matutina, hiperfagia vespertina ou noturna e insônia ${ }^{45}$. Esta síndrome ocorre em resposta a um estresse circadiano que ocorre primariamente em indivíduos obesos, mas também pode ser verificada em indivíduos com elevado nível de estresse, que quando reduzido acarreta melhoria nos sintomas da síndrome. Esse transtorno está associado à obesidade, depressão, baixa auto-estima e redução da fome diurna ${ }^{41}$.

A obesidade está relacionada a fatores psicológicos como o controle, a percepção desi, a ansiedade e 0 desenvolvimento emocional ${ }^{8,41}$. Entretanto ser gordo não significa ter problemas psicológicos, sendo necessário desmistificar esta crença queémuito utilizada como explicação para 0 excesso de peso ${ }^{41}$. Frente a isso, a inclusão dos profissionais da área da psicologia e da psiquiatria na terapêutica da obesidade mostra-se de fundamental importância, por permitir um diagnóstico mais apurado desses aspectos na con- 
formação da obesidade, assim como para a definição do tratamento mais adequado.

Obesidade e estilo de vida moderno: as implicações da dieta e da atividadefísica

0 quadro de balanço energético positivo, que favorece o excesso de peso, tem sido relacionado às mudanças no consumo alimentar, com aumento do fornecimento de energia pela dieta, e redução da atividade física ${ }^{4,5,11,14}$, configurando o estilo de vida ocidental contemporâneo ${ }^{4,5,9,11}$.

0 aumento da ingestão energética pode ser consequência da elevação quantitativa do consumo de alimentos $s^{46-48}$ ou de mudanças qualitativas na dieta, no que concerne ao maior consumo de alimentos com el evada densidade energé tica ${ }^{46,48,49}$, associados ao hábito cada vez mais frequente de comer fora da casa 5 . 0 crescimento da densidade energética da dieta tem sido atribuído ao processo de industrialização dos alimentos, que tem sido verificado na maior parte do 0 cidente ${ }^{5,50}$.

M endonça e Anjos ${ }^{51}$ e Anjos afirmam que existem vários fatores associados à dieta que poderiam contribuir para a elevação do excesso de peso dos brasileiros ao ocasionarem mudanças nos padrões alimentares tradicionais, que são a migração interna, a alimentação fora de casa, 0 crescimento na oferta de fast food e a ampliação do uso de alimentos industrializados. Segundo esses autores, estes aspectos estão diretamente vinculados à renda das famílias e às possibilidades de gasto com alimentação, que está associada ao valor sociocultural dos alimentos em cada grupo social.

Dados da POF ${ }^{12}$ revelam que a média nacional de disponibilidade de alimentos por domicílio éde $1.800 \mathrm{Kcal} / \mathrm{pessoa} / \mathrm{dia}$, sendo $1.700 \mathrm{~K} \mathrm{cal} /$ pessoa/dia no meio urbano e $2.400 \mathrm{Kcal} /$ pessoa/ dia no meio rural. Deve-se ressal tar que a menor disponibilidade calórica no meio urbano possivelmenter refleteuma maior frequência de consumo de alimentos fora do domicílio, de difícil quantificação, e menores necessidades energéticas do que no meio rural.

A tendência de evolução dos padrões de consumo alimentar no período de 1974-1975 a 20022003, passível deestudos apenas nas áreas metropolitanas do país, indica menor contribuição dos carboidratos na dieta embora, haja persistência de um teor excessivo de açúcar na dieta, com redução no consumo de açúcar refinado e incremento no consumo de refrigerantes, e o aumento do aporte de gorduras em geral e gorduras saturadas, não evidenciando qualquer tendência de superação dos níveis insuficientes de consumo de frutas ehortaliças. N ota-se ainda que hábitos tradicionais na dieta do brasileiro, como o arroz e 0 feijão, perderam importância no período, enquanto o consumo de produtos industrializados, como biscoitos, refrigerantes e refeições prontas, aumentou consideravelmente ${ }^{12}$.

Do gasto energético deum indivíduo, faz parte a atividade física realizada por este, que compreende todas as atividades voluntárias, como as ocupacionais, de lazer, domésticas e de deslocamento ${ }^{5,11,48,51}$. A relação da ascensão da obesidade com a redução do nível de atividade física refere-se às mudanças na distribuição das ocupações por setores enos processos de trabalho com redução do esforço físico ocupacional; modificações nas atividades de lazer, que passaram de atividades de elevado gasto energético, como práticas esportivas, para prolongados períodos diante da televisão ou computador; da utilização crescente de equipamentos domésticos com redução do gasto energético da atividade, como, por exemplo, lavar roupa à máquina no lugar de fazêlo manualmente ${ }^{5,48}$; e do uso do automóvel, veículo automotivo para deslocamento ${ }^{48,51}$. Todas essas modificações contribuem para o aumento do comportamento sedentário que desempenha uma função importante no desenvolvimento da obesidade ${ }^{5,48,51,52}$.

Segundo Nunomura ${ }^{53}$, a maioria dos brasileiros não tem o hábito de praticar nenhuma atividadefísica desportiva regular. M ais recentemente, M onteiro ${ }^{49}$ revelou que $68 \%$ dos adultos que vivem na cidade de São Paulo são sedentários. No estudo de Giganteet al. ${ }^{54}$, realizado no município de Pelotas (RS), constatou-se que apenas $32 \%$ da população adulta praticavam exercícios físicos nas horas de lazer.

Deacordo com a literatura, o hábito de assistir televisão por várias horas diárias é considerado um indicador de sedentarismo ${ }^{51,52,55}$, por ser um hábito que diminui a prática esportiva de lazer e aumenta o consumo energético, principalmenteem crianças eadolescentes. Deve-seressaltar que a televisão contribui para a delimitação do estilo de vida ocidental, que inclui os hábitos alimentares, por intermédio do incentivo ao consumo de alimentos difundidos pela propaganda e o marketing $39,51,52$.

Almeida et al. ${ }^{52}$ ressaltam que 0 anúncio de produtos alimentícios ocorreem todos os períodos do dia e que a maioria dos alimentos veiculados possui el evados índices de gorduras, óleos, 
açúcares e sal, o que está em descordo com uma dieta saudável e balanceada eque, portanto, possibilita o excesso de peso. Segundo Serra e Santo ${ }^{39}$, a mídia desempenha papel estruturador na construção e desconstrução das práticas alimentares como, por exemplo, o consumo el evado de fast foods verificado atual mente eque constantementetem sido veiculado pela mídia.

Estudos sobre o padrão de atividade física da população são escassos, já que não existe um estudo de base populacional quetenha investigado o nível de atividadefísica da populaçãa $0^{5,51}$, o que dificulta uma explicação mais consistente sobre a relação desta com a obesidade.

Reconhecidamente, dieta eatividade física são el ementos envolvidos na etiologia da obesidade. Nesta direção, para a Organização Mundial de Saúde ${ }^{1}$ e o M inistério da Saúde ${ }^{8}$ no Brasil, a dieta ocidental e o sedentarismo são os principais fatores de risco para o desenvolvimento da obesidade atualmente nas sociedades modernas.

\section{Consideraçõesfinais}

Frente ao exposto, acreditamos ser necessário criar novas agendas de investigação em saúde e nutrição que valorizem abordagens metodológicas que partam da perspectiva da obesidade enquanto uma enfermidademultifatorial, não-fragmentada, como normalmentese apresenta a literatura sobre o tema. N este sentido, al guns autores já têm realizado, ainda que timidamente, estudos com esta abordagem mais abrangente e plural. A nosso ver, é a partir da construção de estudos dessa natureza que será possível promover uma instrumentalização mais eficiente dos profissionais de saúde para o tratamento da obesidade no nível individual e familiar, no qual as abordagens multidisciplinares e intersetoriais assumiriam destaque.

Ações no âmbito coletivo devem envolver políticas públicas que promovam a saúde, o bemestar ea qual idade de vida das populações ondea parceria entre o governo e a sociedade civil seria um caminho bastante promissor na prevenção e tratamento da obesidade, por meio da responsabilização e do autocuidado, permitindo que a comunidade participe do processo de promoção da saúde.

\section{Colaboradores}

EN Wanderley eVA Ferreira participaram igualmente de todas as etapas da elaboração do artigo. 


\section{Referências}

1. World Health Organization. O besity: Preventing and managing the global epidemic. Report of a WHO Consultation on O besity. Geneva: WHO; 1998.

2. Monteiro CA, Conde $W L$. A tendência secular da obesidade segundo estratos sociais: nordeste e sudeste do Brasil, 1975-1989-1997. Arq Bras Endocrinol M etabol 1999; 43(3):186-194.

3. Pereira LO, Francischi RP, Lancha-Junior HA. Obesidade: hábitos nutricionais, sedentarismo e resistência à insulina. Arq Bras Endocrinol M etabol [periódico na Internet] 2003 [acessado 2007 jan 19]; 47(2):[cerca de 17 p.]. Disponível em: http:// www.scielo.br/pdf/abem/v47n2/a03v47n2.pdf

4. Kac G, Velásquez-M eléndez G. A transição nutricional e a epidemiologia da obesidade na América Latina. Cad Saude Publica 2003; 19(1):4-5.

5. Anjos, LA dos. Obesidade e saúde pública. Rio de Janeiro: Fiocruz; 2006.

6. Associação Brasileira para o Estudo da Obesidade e Síndrome M etabólica. Documento do Consenso Latino- Americano em Obesidade. São Paulo: ABESO; 2001.

7. Pena M, Bacallao J. La obesidad en la pobreza: un problema emergente en las Américas. In: Peña $M$, Bacallao J, editores. La obesidad en la pobreza: un nuevo reto para la salud pública. [Publicação científica $n^{\circ}$ 576]. Washington, D.C.: OPAS; 2000.

8. Brasil. Ministério da Saúde. Secretaria de Atenção à Saúde. Departamento de Atenção Básica. O besidade. Brasília: M inistério da Saúde; 2006.

9. Mondini L, M onteiro CA. Relevância epidemiológica da desnutrição e da obesidade em distintas classes sociais: métodos de estudo e aplicação à população brasileira. Rev. bras. epidemiol. 1998; 1:28-39.

10. Stunkard AJ. Factores determinantes de la obesidad: opinión actual. In: Peña M, Bacallao J, editores. La obesidad en la pobreza: un nuevo reto para la salud pública. [Publicação científica $n^{\circ}$ 576]. Washington, D.C.: OPAS; 2000.

11. Sichieri R. M edidas e determinantes da obesidade. In: Sichieri R, organizadora. Epidemiologia da obesidade. Rio de Janeiro: EDUERJ; 1998.

12. Instituto Brasileiro de Geografia e Estatística. Pesquisa de orçamentos familiares 2002-2003: análise da disponibilidade domiciliar de alimentos e do estado nutricional no Brasil. Rio de Janeiro: IBGE; 2004.

13. Batista Filho M, Rissin A. Nutritional transition in Brazil: geographic and temporal trends. Cad Saude Publica 2003; 19(1):181-191.

14. M onteiro CA, M ondini L, Souza ALM, Popkin BM. Da desnutrição para a obesidade - a transição nutricional no Brasil. In: Monteiro CA, organizador Velhos e novos males da Saúde Pública no Brasil: a evolução do país e de suas doenças. São Paulo: Hucitec; 1995.

15. Popkin BM. Nutritional patterns and transitions. Population and Development. Nutr Rev 1993; 19(1):138-157.

16. Fischler C. Gastro-nomia e gastroanomia: sabedoria do corpo e crise biocultural da alimentação contemporânea. In: Contreras J, organizador. Alimentación y Cultura: Necessidades, Custos y Costumbres. Ciences Humanes i Sociales. Barcelona: Universitat de Barcelona; 1995.
17. Sichieri R, Coitinho DC, Pereira RA, M arins VMR, Moura AS. Variações temporais do estado nutricional e do consumo alimentar no Brasil. Rev. Saude Coletiva 1997; 7 (2):31-50.

18. Escoda M do SQ. Para a crítica da transição nutricional. Cien Saude Colet 2002; 7(2):219-226.

19. Velloso LA. O controle hipotalâmico da fome e da termogênese implicações no desenvolvimento da obesidade. Arq Bras Endocrinol M etabol [periódico na Internet] 2006 [acessado 2006 jul 27]; 50(2):[cerca de 12 p.]. Disponível em: http://www.scielo.br/ scielo.php?pid=S0004-27302006000200003\&script $=$ sci_arttext

20. Bouchard C. Etiology of obesity: genetic factors. Archivos Latinoamericanos de Nutrición 1992; 42(3):127-130.

21. Francischi RPP, Pereira LO, Freitas C S, Klopfer $M$, Santos RC, Vieira P, Lancha Júnior AH. O besidade: atualização sobre sua etiologia, morbidade e tratamento. Rev. Nutr. [periódico na Internet] 2000 [acessado 2006 jul 27]; 13(1):[cerca de 12 p.]. Disponível em: http://www.scielo.br/scielo.php?script=sci arttext\&pid=S1415-52732000000100003\&tlng=en \& Ing=en $\& \mathrm{nrm}=\mathrm{iso}$

22. Silva DO da. 0 fiel da balança na história do corpo obeso em mulheres de baixa renda [dissertação]. Rio de Janeiro (RJ): Escola Nacional de Saúde Pública, Fundação Oswaldo Cruz; 1997.

23. $M$ arques-Lopes I, M arti $A$, M oreno-Aliaga $M J$, $M$ artinez A. Aspectos genéticos da obesidade. Rev. Nutr. [periódico na Internet] 2004 [acessado 2006 jul 27]; 19(1):[cerca de 12 p.]. Disponível em: http:/ /www.scielo.br/scielo.php?script=sci_arttext\&pid $=$ S1415-52732004000300006

24. Duarte ACG, Faillace GBD, Wadi MT, Pinheiro RL. Síndrome M etabólica - Semiologia, Bioquímica e Prescrição Nutricional. São Paulo: Axcel Books do Brasil Editora Ltda; 2005.

25. Claudino AM, Zanella MT, organizadoras. Guia de transtornos alimentares e obesidade. Barueri: $M$ anole; 2005.

26. Guilá MVM. Obesidad: interrelación genética ambiental. Rev. M éd. Clín. Condes [periódico na Internet] 2003 [acessado 2006 jul 27]; 14(3):[cerca de 6 p.]. Disponível em: http://www.clinicalascondes.cl/ Area_Academica/Revista_M edica_Julio_2003/ articulo_002.htm

27. Fonseca-Alaniz M H, Takada J, Alonso-Vale MIC, Lima FB. $O$ tecido adiposo como centro regulador do metabolismo. Arq Bras Endocrinol M etabol [periódico na Internet] 2006 [acessado 2006 jul 27]; 50(2):[cerca de 14 p.] Disponível em: http:// www.scielo.br/pdf/abem/v50n2/29305.pdf

28. Ferreira VA, Magalhães R. O besidade e pobreza: 0 aparente paradoxo. Um estudo com mulheres da Favela da Rocinha. Cad Saude Publica [periódico na Internet] 2005 [acessado 2006 ago 3]; 21(6):[cerca de 9 p.]. Disponível em: http://www.scielo.br/ $\mathrm{pdf} / \mathrm{csp} / \mathrm{v} 21 \mathrm{n6} / 17 . \mathrm{pdf}$ 
29. Brown PJ, Konner M. An anthropological perspective on obesity. In: Goodman AH, Dufour DL, Pelto GH, editors. Nutritional Anthropology - Biocultural Perspectives on Food and Nutrition. California: Mayfield Publishing Company Mountain View; 1999.

30. Fischler $\mathrm{C}$. Obeso Benigno Obeso Maligno. In: Sant'Anna DB, organizadora. Políticas do Corpo: ele mentos para uma história das práticas corporais. São Paulo: Estação Liberdade; 1989.

31. Santos LAS. O corpo, o comer e a comida: um estudo sobre as práticas corporais e alimentares cotidianas a partir da cidade de Salvador - Bahia [dissertação]. São Paulo (SP): Pontifícia Universidade Católica de São Paulo; 2006.

32. M erleau-Ponty M. Fenomenologia da percepção. São Paulo: Martins Fortes; 1994.

33. M auss M. Sociologia e Antropologia. São Paulo: EPU; 1974.

34. Baudrillard J. A sociedade de consumo. Lisboa: Edições 70; Rio de Janeiro: Elfos Editora; 1995.

35. Carvalho MC, Martins A. A obesidade como objeto complexo: uma abordagem filosófico-conceitual. Cien Saude Colet 2004; 9(4):1003-1012.

36. Segal A. Aspectos psiquiátricos da obesidade. ABESO - Órgão Informativo da Associação Brasileira para o Estudo da O besidade [periódico na Internet] 2003 [acessado 2006 ago 3];12:[cerca de 3 p.]. Disponível em: http://www.abeso.org.br/revista/revista12/ aspectos.htm

37. Almeida GAN, Santos JE, Pasian SR, Loureiro SR. Percepção de tamanho e forma corporal de mulheres: estudo exploratório. Psicologia em Estudo 2005; 10(1):27-35.

38. Lins APM. Fatores associados ao sobrepeso em muIheres de 20 a 59 anos no M unicípio do Rio de Janeiro [dissertação]. Rio de Janeiro (RJ): Fiocruz; 1999.

39. Serra GM A, Santos EM. Saúde e mídia na construção da obesidade e do corpo perfeito. Cien Saude Colet [periódico na Internet] 2003 [acessado 2007 jan 19]; 8(3):[cerca de 11 p]. Disponível em: http:/ /www.scielo.br/pdf/csc/v8n3/17450.pdf

40. M atos MIR, Zanella MT. Alterações do comportamento alimentar, ansiedade, depressão e imagem corporal em pacientes com obesidade grau III. ABESO - Órgão Informativo da Associação Brasileira para o Estudo da Obesidade [periódico na Internet] [acessado 2006 ago 3]. Disponível em: http:// www.abeso.org.br/revista/revista9/alteracoes.htm

41. Dobrow IJ, Kamenetz C, Devlin MJ. Aspectos psiquiátricos da obesidade. Rev Bras Psiquiatr [periódico na Internet] 2002 [acessado 2006 jul 28]; 24(3)[cerca de 5 p.]. Disponível em: http://www. scielo.br/pdf/rbp/v24s3/13975.pdf

42. Stunkard AJ. Eating patterns and obesity. Psychiatr Q 1959; 33:284-295.

43. Appolinário JC. Transtorno da compulsão alimentar periódica: uma entidade clínica emergente que responde ao tratamento farmacológico. Rev Bras Psiquiatr [periódico na Internet] 2004 [acessado 2007 jan 19]; 26(2):[cerca de 5 p.]. Disponível em: http://www.scielo.br/scielo.php?script =sci 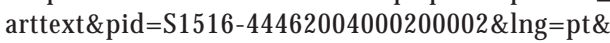 $\mathrm{nrm}=\mathrm{i}$ so\&tlng=pt
44. Bernardi F, Cichelero C, Vitolo MR. Comportamento de restrição alimentar e obesidade. Rev. Nutr. [periódico na Internet] 2005 [acessado 2007 jan 19]; 18(1): ):[cerca de 9 p.]. Disponível em: http:// www.scielo.br/scielo.php?script=sci_arttext $\&$ pid $=$ S1415-52732005000100008

45. Stunkard AJ, Grace WJ, Wolff HG. The night-eating syndrome. Am J M ed 1955; 19:78-86.

46. Mondini $L, M$ onteiro $C A$. Mudanças no padrão de alimentação da população urbana brasileira (19621988). Rev. Saude Publica 1994; 28(6):433-439.

47. Monteiro CA, M ondini L, Costa R. Mudanças na composição e adequação nutricional da dieta familiar nas áreas metropolitanas do Brasil (19881996). Rev. Saude Publica 2000; 34:251-258.

48. Organización Mundial De La Salud/Organización Panamericana De La Salud. Obesidad, alimentación y actividad física. In: OM S/OPAS. 37a Sesión Del Subcomité de Planificación y Programación del Comité Ejecutivo. Washington, D.C.: OM S/OPAS; 2003.

49. M onteiro CA. Epidemia: obesidade já é o segundo maior fator de risco na cidade. Folha de São Paulo 2004; 14 mar.

50. Bleil SI. O padrão alimentar ocidental: considerações sobre as mudanças de hábitos no Brasil. Cadernos de Debate 1998; 1:1-25.

51. Mendonça $\mathrm{CP}$, Anjos LA dos. Aspectos das práticas alimentares e da atividade física como determinantes do crescimento do sobrepeso/obesidade no Brasil. Cad Saude Publica 2004; 20(3):698-709.

52. Almeida SS, Nascimento PCBD, Quaioti TCB. Quantidade e qualidade dos produtos alimentícios anunciados na televisão brasileira. Rev. Saude Publica [periódico na Internet] 2002 [acessado 2006 ago ]; 36(3):[cerca de 3 p.]. Disponível em: http:// www.scielo.br/pdf/rsp/v36n3/10500.pdf.

53. N unomura M. Motivos de adesão à atividade física em função das variáveis idade, sexo, grau de instrução e tempo de permanência. Rev Brasileira de Atividade Física e Saúde 1998; 3(3):45-58.

54. Gigante DP, Barros FC, Post CLA, Olinto MTA. Prevalência de obesidade em adultos e seus fatores de risco. Rev. Saude Publica 1997; 31(3):236-246.

55. Frutuoso MFP, Bismarck-Nasr EM, Gambardella AM D. Redução do dispêndio energético e excesso de peso corporal em adolescentes. Rev. Nutr. 2003; 16 (3):257-263.

Artigo apresentado em 28/19/2007

Versão final apresentada em 13/12/2007 